\title{
Gaussian transformations and distillation of entangled Gaussian states
}

\author{
Jaromír Fiurášek \\ Department of Optics, Palacký University, 17. listopadu 50, 77200 Olomouc, Czech Republic
}

\begin{abstract}
We prove that it is impossible to distill more entanglement from a single copy of a two-mode bipartite entangled Gaussian state via LOCC Gaussian operations. More generally, we show that any hypothetical distillation protocol for Gaussian states involving only Gaussian operations would be a deterministic protocol. Finally, we argue that the protocol considered by Eisert et al. quantph/0204052 is the optimum Gaussian distillation protocol for two copies of entangled Gaussian states.

PACS numbers: 03.67.-a, 42.50.Dv
\end{abstract}

Quantum entanglement is a key ingredient of many protocols for quantum information processing such as quantum teleportation [1] or quantum cryptography [2]. Usually, the entangled particles are distributed among two distant parties traditionally called Alice and Bob. In practice, the transmission channel used for this distribution is always noisy and imperfect, which prevents Alice and Bob from sharing a maximally entangled state even if Alice can prepare such state locally in her lab. Fortunately, the errors introduced by noisy quantum channels can be overcome by the so-called entanglement distillation protocols, by which Alice and Bob can extract from a large number of weakly entangled mixed states a smaller number of highly entangled almost pure states [3, 4.

Recently, a great deal of attention has been devoted to the quantum information processing with continuous quantum variables and continuous-variable analogues of various protocols developed originally in the framework of discrete quantum variables have been established. Remarkably, linear optics, parametric amplifiers, and homodyne detectors suffice for implementation of many of these protocols including continuous-variable teleportation [5], cryptography [6], and cloning [7]. However, one important protocol missing in our toolbox is a feasible distillation protocol for continuous variables. We are particularly interested in distillation protocols for entangled Gaussian states because these states can easily be generated in the laboratory. The protocols suggested so far involve rather complicated nonlinear transformations such as subtraction of a single photon [8 or quantum non-demolition measurement of total photon number in several modes [9]. It would be of great help to have a distillation protocol for continuous variables that could be implemented with linear optics and which would distill Gaussian entangled states. However, no such protocol is currently known and it is an open question whether such distillation protocol exists at all.

In the present paper we attempt to shed some light on this issue making use of the formalism of Gaussian completely positive (CP) maps [10, 11]. These maps represent all transformations that can be carried out with the help of passive and active linear optical elements, homodyne detectors, and auxiliary optical modes prepared initially in Gaussian states. These transformations may be deterministic or probabilistic. In the latter case we accept or reject the output state in dependence on the output of a quantum measurement (with some Gaussian probability distribution). We shall consider an arbitrary bipartite probabilistic Gaussian operation which can be implemented with the help of local operations and classical communication (LOCC). We shall prove that for input bipartite Gaussian states it is always possible to construct a deterministic LOCC Gaussian transformation that yields the same output state (for a fixed input) as a given probabilistic LOCC Gaussian transformation. This implies that it is impossible to distill more entanglement from a single copy of entangled Gaussian state by means of Gaussian operations. This should be contrasted with distillation protocols for a single copy of two-qubit entangled state where the LOCC operations may in some cases allow to extract more entanglement [18. In particular, any pure entangled two-qubit state can be transformed with certain probability via LOCC operations onto maximally entangled Bell state. Furthermore, our results imply that any hypothetical Gaussian distillation protocol optimized for given shared entangled Gaussian states would be a deterministic protocol and we shall find a generic structure of this optimum protocol. A version of this optimum protocol where Alice and Bob share two identical copies of Gaussian state with symmetric covariance matrix has been considered by Eisert et al. [12] who employed the log-negativity as the entanglement measure and proved that it is impossible to distill entanglement via this protocol. These findings thus strongly support the conjecture that it is impossible to distill entangled Gaussian states via Gaussian operations and that some nonlinearity is necessary.

We shall extensively exploit the Jamiolkowski isomorphism 13 between completely positive maps $\mathcal{M}$ and positive-semidefinite operators (bipartite quantum states) $\chi$ on tensor product of input and output Hilbert spaces $\mathcal{H} \otimes \mathcal{K}$. In terms of the operator $\chi \geq 0$ the relation between input and output density matrices can be written as a partial trace over the input space,

$$
\rho_{\text {out }}=\operatorname{Tr}_{\text {in }}\left[\chi \rho_{\text {in }}^{T} \otimes \mathbb{1}_{\text {out }}\right]
$$


where $T$ stands for the transposition in some fixed basis and $\mathbb{1}_{\text {out }}$ denotes an identity operator on output space. The operator $\chi$ can be obtained from a maximally entangled state on $\mathcal{H}^{\otimes 2},|\psi\rangle=\sum_{j=1}^{d}|j\rangle_{1}|j\rangle_{2},(d=\operatorname{dim} \mathcal{H})$ if the map $\mathcal{M}$ is applied to one part of this state,

$$
\chi=\mathcal{M} \otimes \mathcal{I}[|\psi\rangle\langle\psi|] .
$$

Here $\mathcal{I}$ stands for the identity transformation.

In continuous variable systems, we deal with infinite dimensional Hilbert spaces and the maximally entangled state $|\psi\rangle$ becomes a tensor product of $N_{\text {in }}$ (unphysical) two-mode infinitely squeezed vacuum states, where $N_{\text {in }}$ is the number of input modes. Gaussian completely positive maps are defined as maps which transform Gaussian states into Gaussian states. Gaussian CP maps are thus isomorphic to bipartite Gaussian quantum states $\chi$. Now any Gaussian state $\chi$ is completely characterized by the first and second moments: mean values of quadratures and a covariance matrix $\Gamma$. Define vector of quadratures $\vec{r}=\left(x_{1}, p_{1}, \ldots, x_{N}, p_{N}\right)^{T}$ where $N$ is the total number of input+output modes. The elements of matrix $\Gamma$ are defined as $\Gamma_{i j}=\left\langle\Delta r_{i} \Delta r_{j}\right\rangle+\left\langle\Delta r_{j} \Delta r_{i}\right\rangle$, where $\Delta r_{i}=r_{i}-\left\langle r_{i}\right\rangle$. Nonzero mean values of the quadratures of the Gaussian state $\chi$ representing a Gaussian CP map indicate that this map involves certain displacements. However, these operations can be performed locally and are therefore irrelevant for the entanglement properties and can be omitted. Thus we can assume that $\left\langle r_{i}\right\rangle=0$ and the CP map $\chi$ is fully described by the covariance matrix $\Gamma$. It is convenient to split the matrix $\Gamma$ into input and output parts and write

$$
\Gamma=\left(\begin{array}{cc}
A & C \\
C^{T} & B
\end{array}\right),
$$

where $A$ stands for the covariance matrix of the "input" modes, $B$ is the covariance matrix of "output" modes and $C$ contains the input-output correlations. The input-output transformation (11) rewritten in terms of the Wigner functions reads

$$
W_{\text {out }}\left(\vec{r}_{\text {out }}\right)=(2 \pi)^{N_{\text {in }}} \int_{-\infty}^{\infty} W_{\chi}\left(\vec{r}_{\text {in }}, \vec{r}_{\text {out }}\right) W_{\text {in }}\left(R \vec{r}_{\text {in }}\right) d \vec{r}_{\text {in }},
$$

where $R=\operatorname{diag}(1,-1,1,-1, \ldots, 1,-1)$ is a diagonal matrix which represents the transposition in phase space $\left(x_{j} \rightarrow x_{j}, p_{j} \rightarrow-p_{j}\right)$. It is convenient to deal with characteristic functions which are Fourier transforms of the Wigner functions,

$$
\mathcal{C}(\vec{q})=\int_{-\infty}^{\infty} W(\vec{r}) \exp (i \vec{r} \cdot \vec{q}) d \vec{r}
$$

On expressing all Wigner functions in terms of the characteristic functions, we obtain from Eq. (何),

$$
\mathcal{C}\left(\vec{q}_{\text {out }}\right)=(2 \pi)^{-N_{\text {in }}} \int_{-\infty}^{\infty} \mathcal{C}_{\chi}\left(\vec{q}_{\text {in }}, \vec{q}_{\text {out }}\right) \mathcal{C}_{\text {in }}\left(-R \vec{q}_{\text {in }}\right) d \vec{q}_{\text {in }} .
$$

Assuming input Gaussian state with covariance matrix $\Gamma_{\text {in }}, \mathcal{C}_{\text {in }}(\vec{q})=\exp \left(-\frac{1}{4} \vec{q}^{T} \Gamma_{\text {in }} \vec{q}\right)$, we find that the the output state is also Gaussian with covariance matrix given by

$$
\Gamma_{\text {out }}=B-C^{T}\left(A+R \Gamma_{\text {in }} R\right)^{-1} C .
$$

We now prove a very important feature of Gaussian $\mathrm{CP}$ maps. It holds that for every input Gaussian state and a probabilistic (trace-decreasing) LOCC Gaussian $\mathrm{CP}$ map there exists a deterministic (trace-preserving) LOCC Gaussian CP map which transforms the input state into the output state with the covariance matrix (7). The explicit construction of the trace-preserving map is inspired by recent works on the possibility of storing quantum dynamics in quantum states 14, 15. The basic strategy is to encode the transformation into a bipartite state $\chi$ which then serves as a quantum channel in the teleportation. In this way, the desired transformation is carried out with certain probability depending on the dimension of the Hilbert space.

The continuous-variable analogue of this scheme goes as follows. We prepare a Gaussian state $\chi$ with covariance matrix $\Gamma$ given by Eq. (3) and carry out a Bell measurement on the input state and the input modes of the state $\chi$. This measurement is performed separately for each corresponding pair of modes and consists of measuring the difference of $x$ quadratures and sum of $p$ quadratures by means of homodyne detectors. Let the vectors $\vec{x}_{d}$ and $\vec{p}_{d}$ contain the measurement outcomes for $x$ and $p$ quadratures, respectively. The (non-normalized) Wigner function of the output modes conditioned on the measurement outcome $\vec{r}_{d}$ reads

$$
\begin{aligned}
W_{\text {out }}\left(\vec{r}_{\text {out }} \mid \vec{r}_{d}\right)= & \int_{-\infty}^{\infty} W_{\chi}\left(\vec{r}_{\text {in }}, \vec{r}_{\text {out }}\right) W_{\text {in }}(\vec{r}) \delta\left(\vec{x}_{\text {in }}-\vec{x}-\vec{x}_{d}\right) \\
& \times \delta\left(\vec{p}_{\text {in }}+\vec{p}-\vec{p}_{d}\right) d \vec{x}_{\text {in }} d \vec{p}_{\text {in }} d \vec{x} d \vec{p} .
\end{aligned}
$$

Consider input Gaussian state. It turns out that the covariance matrix of the output state is given by Eq. (7) and does not depend on the measured quadratures $\vec{x}_{d}$ and $\vec{p}_{d}$. However, the output state is displaced by

$$
\vec{r}_{\text {cond }}=C^{T}\left(A+R \Gamma_{\text {in }} R\right)^{-1} \vec{r}_{d} .
$$

If we know the input state and the transformation, then we can calculate $\vec{r}_{\text {cond }}$ for given detected quadratures $\vec{x}_{d}$, $\vec{p}_{d}$ and by means of suitable displacement transformation applied to output state we can always set the coherent signal in the output state to zero. In this way we obtain in a deterministic manner an output state which has the covariance matrix (7). This procedure is essentially the Braunstein-Kimble scheme for teleportation of continuous variables [5]. We should note here that many Gaussian CP maps, in particular all trace-preserving maps, are represented by unphysical (infinitely squeezed) states $\chi$. However, we can approximate such unphysical state by a 


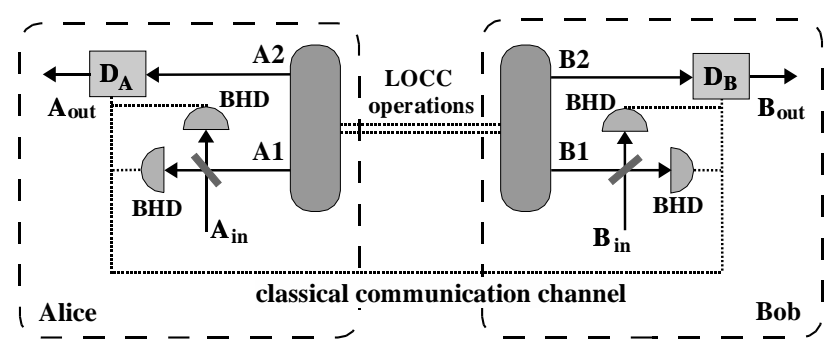

FIG. 1: Setup for implementation of deterministic LOCC Gaussian CP map that is for certain input Gaussian state equivalent to a given probabilistic LOCC Gaussian CP map.

physical finitely squeezed state with an arbitrarily high accuracy and thus also approximate the transformation (7) with arbitrarily high precision.

Let us now turn our attention to the LOCC Gaussian CP maps. Obviously, every LOCC Gaussian map $\mathcal{M}_{\text {LOCC }}$ is isomorphic to a Gaussian state $\chi$ which is separable with respect to Alice and Bob. (Note that according to Eq. (2) Alice and Bob can prepare the state $\chi$ in their labs via LOCC operations.) A scheme for deterministic implementation of any LOCC Gaussian CP map (for known input Gaussian state) is shown in Fig. 1 for the simplest case when there is a single input and a single output mode on each side. By means of LOCC operations, Alice and Bob prepare the four-mode state $\chi$ representing the CP map ( $A 1$ and $B 1$ are input modes and $A 2$ and $B 2$ are output modes). Alice mixes her part of the input state in mode $A_{\text {in }}$ with $A 1$ on balanced beam splitter and measures $x_{A 1}-x_{A \text { in }}$ and $p_{A \text { in }}+p_{A 1}$ by means of two balanced homodyne detectors. Bob performs the same operations with his modes $B 1$ and $B_{\text {in }}$. Alice and Bob exchange the results of their measurements via classical communication channel and apply appropriate local displacement transformations to the modes $A 2$ and $B 2$ thereby producing the two-mode output state $A_{\text {out }}$ and $B_{\text {out }}$. Notice that the protocol works only for Gaussian states. If the input state is not Gaussian, than it may happen that some trace-decreasing LOCC Gaussian CP maps will yield outputs that cannot be obtained with any trace-preserving LOCC Gaussian CP map.

A very important implication concerning distillation protocols is that we cannot distill more entanglement from a single copy of two-mode bipartite entangled Gaussian state by means of LOCC Gaussian operations. This follows from the fact that any probabilistic LOCC Gaussian operation can be replaced by a deterministic one which yields the same output (for a given input Gaussian state). However, any reasonable measure of entanglement must be non-increasing under deterministic LOCC operations. Here we note that this impossibility was noted in a paper by Parker et al. [16], who considered distillation via continuous-variable entanglement swapping. While this procedure works for Schrödinger cat states, it fails for two-mode squeezed vacuum. Parker et al. also noticed that in the latter case, the probabilistic Gaussian transformation becomes deterministic. Our arguments show that this is a general feature of Gaussian states and Gaussian CP maps.

Let us have a more detailed look at the structure of bipartite LOCC Gaussian CP maps. It was shown by Werner and Wolf [17 that a bipartite Gaussian state is separable if and only if it can be transformed via local symplectic transformations into state with positive Glauber-Sudarshan representation, i.e. a state which is a convex mixture of coherent states and is not squeezed. We can thus write

$$
\chi_{\mathrm{LOCC}}=\int_{-\infty}^{\infty} P(\alpha, \beta) S_{A}|\alpha\rangle\left\langle\alpha\left|S_{A}^{\dagger} \otimes S_{B}\right| \beta\right\rangle\langle\beta| S_{B}^{\dagger} d \alpha d \beta,
$$

where $P(\alpha, \beta) \geq 0$ is classical Gaussian probability distribution and $|\alpha\rangle,|\beta\rangle$ denote (multimode) coherent states of Alice's and Bob's modes. We have seen that it is impossible to distill a single copy of a Gaussian state by means of LOCC Gaussian transformations. What if Alice and Bob posses several copies? Assume that Alice and Bob apply the LOCC Gaussian map (9) to their states. This distillation map takes all copies as an input and yields a single copy of two-mode state shared by Alice and Bob as the output. The output state is a mixture of states with identical covariance matrices and varying displacements. In terms of Wigner functions, we can write

$$
W_{\text {out }}(\vec{r})=\int_{-\infty}^{\infty} \tilde{P}(\alpha, \beta) W\left(\vec{r}-\vec{r}_{d}(\alpha, \beta)\right) d \alpha d \beta,
$$

where $\vec{r}_{d}(\alpha, \beta)$ is the displacement and $\tilde{P}(\alpha, \beta) \geq 0$. However, all states with Wigner functions $W\left(\vec{r}-\vec{r}_{d}\right)$ exhibit the same entanglement, because entanglement depends only on the covariance matrix and not on the displacement. Hence it is always optimum to choose an LOCC CP map which is represented by a pure Gaussian state. An analogous situation arises in distillation of a single pair of entangled qubits where it is optimum to apply local filtering operation [18] (this is a tracedecreasing CP map whose Kraus decomposition contains only one term and the CP map is thus represented by a pure state).

Since we assume that we know the state that we want to distill, we can transform any LOCC trace-decreasing map onto trace-preserving map hence the optimum protocol will be deterministic. Consider now the simplest case when Alice and Bob share two pairs of entangled Gaussian states. The transformation on Alice's side is represented by a pure three-mode Gaussian state, which can be obtained from three-mode vacuum via some threemode symplectic transformation. This three-mode state splits into two input modes and one output mode. By means of "local" symplectic transformations on input and output modes, we can transform the covariance matrix 


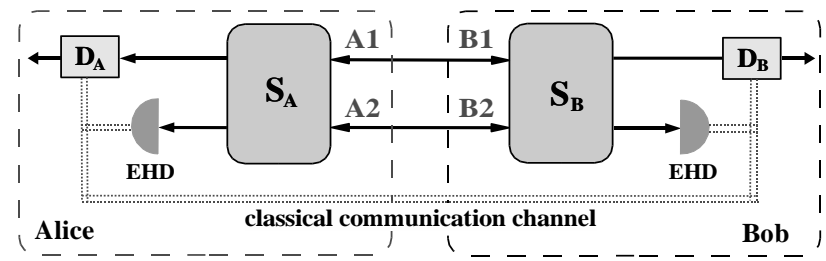

FIG. 2: Optimal distillation protocol for two copies of twomode entangled Gaussian states.

of this state into the form

$$
\Gamma=\left(\begin{array}{cccccc}
a & 0 & 0 & 0 & d_{1} & 0 \\
0 & a & 0 & 0 & 0 & d_{2} \\
0 & 0 & b & 0 & e_{1} & 0 \\
0 & 0 & 0 & b & e_{3} & e_{2} \\
d_{1} & 0 & e_{1} & e_{3} & c & 0 \\
0 & d_{2} & 0 & e_{2} & 0 & c
\end{array}\right)
$$

The reduced density matrix of each input and output mode is density matrix of thermal state and there are no correlations between the two input modes. Since the whole three-mode state is pure, the density matrix of the two input states has the same eigenvalues as the reduced density matrix of the output state. This is possible only if one of the input modes is in pure vacuum state. This leads to $a=c, b=1, e_{1}=e_{2}=e_{3}=0$. One of the input modes is effectively decoupled and we end up with pure two-mode squeezed vacuum state. We thus have a very appealing and intuitive picture: any pure threemode state can be prepared if we start from (suitably chosen) two-mode squeezed vacuum state and a vacuum state and apply single-mode symplectic transformation to the output mode and two-mode symplectic transformation $S_{i}$ to two input modes. We may thus write the transformation on Alice's side in the form

$$
\chi_{A}=S_{i}\left(\chi_{A 0} \otimes|0\rangle\langle 0|\right) S_{i}^{\dagger},
$$

where $\chi_{A 0}$ is an operator on Hilbert space of two modes (one input and one output). To see what are the implications, we insert this expression into formula (11) and for the moment do not consider Bob's states. We get

$$
\begin{aligned}
\rho_{\text {out }} & =\operatorname{Tr}_{\text {in }}\left[\chi_{A 0} \otimes|0\rangle\langle 0| S_{i}^{\dagger} \rho_{\text {in }}^{T} S_{i} \otimes \mathbb{1}_{\text {out }}\right] \\
& =\operatorname{Tr}_{\text {in }}\left[\chi_{A 0} \otimes|0\rangle\langle 0|\left(S_{i}^{T} \rho_{\text {in }} S_{i}^{*}\right)^{T} \otimes \mathbb{1}_{\text {out }}\right] .
\end{aligned}
$$

From this formula we can see that the transformation reduces to the following three steps: (i) apply symplectic transformation $S_{i}^{T}$ to the input two-mode state. (ii) project the second mode onto vacuum state. (iii) Apply a CP map $\chi_{A 0}$ to the first mode. The transformation on Bob's side has the same structure. This protocol can be further simplified. After Alice and Bob project one of modes onto vacuum state, they possess only a single mode each. The application of the local maps $\chi_{A 0}\left(\chi_{B 0}\right)$ cannot increase entanglement, because we have shown that it is impossible to distill a single copy of two-mode entangled Gaussian state by means of LOCC Gaussian operations. Thus we need not consider the transformations $\chi_{A 0}\left(\chi_{B 0}\right)$ and the resulting optimum simplified distillation scheme is shown in Fig. 2. Alice and Bob share two pairs of Gaussian states. Alice's and Bob's modes are labeled as $A 1, A 2$ and $B 1, B 2$, respectively. Both Alice and Bob locally apply some two-mode symplectic transformations $S_{A}$ and $S_{B}$ to their modes. Subsequently they both feed the modes $A 2$ and $B 2$ to eight-port homodyne detectors (EHD) thereby projecting them into coherent states $|\alpha\rangle$ and $|\beta\rangle$. Finally, they exchange the results of their measurements and displace appropriately the output states. This scheme is deterministic and represents the optimum Gaussian distillation protocol for Gaussian states. Eisert et al. proved that it is impossible to distill entanglement from two identical copies of two-mode Gaussian state with symmetrical covariance matrix via this protocol [12. All these results strongly support the conjecture that it is impossible to distill entangled Gaussian states with Gaussian operations.

Note added: After this work was completed, I learned that Giedke and Cirac 19 have also investigated the properties of trace-decreasing Gaussian $\mathrm{CP}$ maps and they independently obtained similar results. Moreover, they proved that the distillation of Gaussian states with Gaussian operations is impossible for an arbitrary number of modes per site.

I would like to thank J. Eisert, P. van Loock, G. Giedke, N. Cerf, R. Filip, and L. Mišta, Jr. for discussions. This work was supported by Grant No LN00A015 of the Czech Ministry of Education and by the EU grant under QIPC, project IST-1999-13071 (QUICOV).

[1] C.H. Bennett et al., Phys. Rev. Lett. 70, 1895 (1993).

[2] A.K. Ekert, Phys. Rev. Lett. 67, 661 (1991).

[3] C.H. Bennett et al., Phys. Rev. Lett. 76, 722 (1996).

[4] D. Deutsch et al., Phys. Rev. Lett. 77, 2818 (1996).

[5] S.L. Braunstein and H.J. Kimble, Phys. Rev. Lett. 80, 869 (1998); A. Furusawa et al., Science 282, 706 (1998).

[6] T.C. Ralph, Phys. Rev. A 61, 010303(R) (2000); F. Grosshans and P. Grangier, Phys. Rev. Lett. 88, 057902 (2002); Ch. Silberhorn et al., ibid. 88, 167902 (2002).

[7] S.L. Braunstein et al., Phys. Rev. Lett. 86, 4938 (2001); J. Fiurášek, Phys. Rev. Lett. 86, 4942 (2001).

[8] T. Opatrný et al., Phys. Rev. A 61, 032302 (2000).

[9] L.M. Duan et al., Phys. Rev. Lett. 84, 4002 (2000).

[10] G. Lindblad, J. Phys. A: Math. Gen. 33, 5059 (2000).

[11] J. Eisert and M. B. Plenio, quant-ph/0109126.

[12] J. Eisert, S. Scheel, and M.B. Plenio, quant-ph/0204052.

[13] A. Jamiolkowski, Rep. Math. Phys. 3, 275 (1972).

[14] W. Dür and J.I. Cirac, Phys. Rev. A 64, 012317 (2001)

[15] M. Hillery et al., Phys. Rev. A 65, 022301 (2002).

[16] S. Parker et al., Phys. Rev. A 61, 032305 (2000).

[17] R.F. Werner et al., Phys. Rev. Lett. 86, 3658 (2001).

[18] A. Kent et al., Phys. Rev. Lett. 83, 2656 (1999).

[19] G. Giedke and J.I. Cirac, quant-ph/0204085. 